\title{
Dynamical instability of laminated plates with external cutout
}

\author{
Jan Awrejcewicz a,b,*, Lidiya $\operatorname{Kurpa}^{c}$, Olga Mazur ${ }^{c}$ \\ a Lodz University of Technology, Department of Automation, Biomechanics and Mechatronics, 1/15 Stefanowski St., 90-924 Lodz, Poland \\ ${ }^{\mathrm{b}}$ Warsaw University of Technology, Department of Vehicles, 84 Narbutta Str., 02-524 Warsaw, Poland

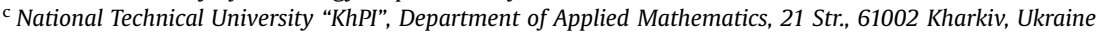

\section{A R T I C L E I N F O}

\section{Article history:}

Received 29 January 2014

Received in revised form

4 January 2016

Accepted 5 January 2016

Available online 12 January 2016

Keywords:

Laminated plates

Parametric vibrations

R-function theory

Instability

\begin{abstract}
A B S T R A C T
A method to study dynamical instability and non-linear parametric vibrations of symmetrically laminated plates of complex shapes and having different cutouts is proposed. The first-order shear deformation theory (FSDT) and the classical plate theory (CPT) are used to formulate a mathematical statement of the given problem. The presence of cutouts essentially complicates the solution of buckling problem, since the stress field is non-uniform. At first, a plane stress analysis is carried out using the variational Ritz method and the R-functions theory. The obtained results are applied to investigate buckling and parametric vibrations of laminated plates. The developed method uses the R-functions theory, and it may be directly employed to study laminated plates of arbitrary forms and different boundary conditions. Besides, the proposed method is numerical-analytical, what greatly facilitates a solution of similar-like non-linear problems. In order to show the advantage of the developed approach, instability zones and response curves for the layered cross- and angle-ply plates with external cutouts are constructed and discussed.
\end{abstract}

(c) 2016 Elsevier Ltd. All rights reserved.

\section{Introduction}

Multilayer plates and shells made of composite materials are widely used in many branches of industry and civil engineering. Usually, laminated composite structures have cutouts which are introduced due to industrial and practical requirements. Note that these cutouts may appear as either internal or external ones. The ways to fix these cutouts are varied and depend on the destination and the location of holes or cutouts. It is well known that, as a rule, cutouts change static and dynamic characteristics of the structures which are subjected to various time-dependent external loads. Therefore, a study of a dynamic behavior of multi-layer elements of thin-walled structures with cutouts is one of important problems arising in engineering design and fabrication.

The described problem has been investigated in numerous publications [1,2,5,6,15-17,19,23,24]. An overview of the references devoted to an analysis of laminated plates with cutouts is given by Anil et al. [2], Datta and Biswas [8], Qatu et al. [19], Sahu and Datta [24,25], Zhang and Yang [26], Chen et al. [27], and others.

Our critical review of the state-of-the-art of the problem reported in the available publications and devoted to the study of

\footnotetext{
* Corresponding author.

E-mail addresses: jan.awrejcewicz@p.lodz.pl (J. Awrejcewicz), L.Kurpa@mail.ru (L. Kurpa), mazuroly@gmail.com (O. Mazur).
}

parametric vibrations of laminated plates with inhomogeneous subcritical states have shown that rectangular plates with a free square or circular central cutout have been mostly considered $[2,6,17,23,24,26]$. Boundary conditions on the outer contour are usually either simply supported or clamped. In order to study the non-linear dynamics of plates with cutouts, the majority of authors suggest applying the finite element method (FEM) $[5,10,18,26]$. It should be noted that there are practically no publications devoted to an investigation of plates having complex shape and various arrangement of holes or cuttings. However, in practice, thin-walled composite elements of different shapes and with various types of cutouts are commonly used. In addition, ways of fastening and loading may be also varied while manufacturing plates of complex shapes. These observations, coming from industrial requirements, yielded the motivation of our paper.

To investigate parametric vibrations of laminated plates of a complex form, original and novel approach has been proposed earlier in our works $[3,12,13]$. The approach has been based on an application of the R-functions theory [22] and variational methods, which makes it applicable to study vibrations of plates with cutouts of different forms and having different boundary conditions. The mentioned method has been developed for thin laminated plates, for which the classical plate theory (CPT) is validated. In addition, the method has been successfully applied to plates under counterbalancing compressive loads. In the present study, the abovementioned approach is generalized for moderately thick laminated plates, parametric vibrations and stability of which can 
be analyzed by the refined theory of the first order (FSDT). We study laminated plates, provided that their subcritical state may be inhomogeneous, i.e. a plate can have external or internal cutouts as well as have different ways of their fastening. Besides, acting loads can balance each other through a clamped part of the boundary. It should be emphasized that, according to the proposed approach, it is necessary to solve a series of linear boundary value problems. These problems are solved by the Ritz method combined with the R-functions theory $[11,22]$. Due to original representation of unknown displacements and application of the Bubnov-Galerkin procedure, the initial non-linear system of PDEs (partial differential equations) governing the plate dynamics is reduced to a non-linear system of ordinary differential equations (ODEs) of a second order. The coefficients of this system are obtained in an analytical form for plates of an arbitrary shape and expressed by double integrals of constructed functions.

It should be emphasized that a numerical realization of the developed algorithm for a multi-mode approximation, and also for solving the obtained non-linear system of ODEs consisting of numerous equations of the second order, belongs to challenging tasks in the case of domains with complex forms. However, for many engineering applications it is sufficient to consider a singlemode approximation, which requires a study of the classical Mathieu non-linear equation. As it has been shown in many studies reported in Refs. [28-36], this equation is used to predict nonlinear phenomena yielded mainly by a parametric resonance. Motivated by this recently revisited application of the non-linear Mathieu equation, our analysis has been restricted to only the first mode approximation. Application of the developed numericalanalytical approach has allowed to construct instability zones as well as to study non-linear vibrations after a loss of stability. The effects of various parameters on the regions of dynamic stability/ instability have been illustrated and discussed using a few computational examples.

On the other hand, the presented results obtained for nonlinear vibrations of laminated plates by means of application of a single mode can be considered as a solution to the problem in the first approximation. In a general case, when a multi-mode interaction plays a crucial role, a convergence study with an increasing number of modes should be carried out. However, the numerical realization of the latter problem is quite complicated in the case of complex geometry domains.

Since our numerical examples are restricted to only a first mode approximation, we briefly describe our motivation for this study, in particular from the point of view of engineering applications. Recently, high interest in manufacturing sensors and actuators using electrically-actuated continuous members like microbeams and microplates (the so-called MEMS devices and systems) is observed in a wide spectrum of mechanical and mechatronics industries. The existing manufacturing techniques and infrastructure of semiconductor industry, introducing new design and ways of testing performance of microbeam-based and microplate-based systems, are mainly aimed at the reduced-order models of simple one-degree-of-freedom lumped-mass torsional or translational systems.

The classical well-known second-order non-linear ODEs are widely applied in the field of MEMS. In the paper by Turner et al. [28], the Mathieu equation has been utilized for modeling dynamics of a swing, the stability of ships and columns and Faraday's surface wave patterns on the water, putting emphasis on parametrically excited torsional oscillators in a single-crystal silicon MEMS system.

A torsional silicon microresonator has been fabricated and studied in Ref. [29]. It consisted of a rectangular paddle symmetrically suspended by narrow beams of $150-200 \mathrm{~nm}$ in width. These metallic layers have been evaporated on the top surface introduce an electric input. Motion of the MEM structure is caused by applying potential between the top and bottom surfaces. A simple second-order ODE has been used to model behavior of continuous systems. In particular, an evidence of parametric amplification has been illustrated and analyzed by modulating the spring constant at twice the resonant frequency.

An auto-parametric amplification-based continuous mass sensor consisting of parallel interdigitated comb finger banks and two sets of non-interdigitated comb fingers on each side, supplemented with four folded beams providing elastic recovery forces, has been modeled, analyzed and experimentally validated by Zhang et al. [30]. The non-linear Mathieu equation has been applied to predict non-linear phenomena exhibited by the main parametric resonance.

The Ruelle-Takens route to chaotic resonance of a nanomechanical device manufactured of three coupled beam resonators excited by magnetomotive driving has been reported by Scheible et al. [31]. The experimentally obtained Fourier spectrum has motivated the authors to apply the standard Duffing equation to study the system chaotic response, although the authors have reported a need to introduce more degrees of freedom.

Zhang and Meng [32] have introduced the simplified mass-springdamping model of an electrostatically actuated micro-cantilever MEMS device. In particular, main and parametric resonances as well as non-linear behaviors, when subjected to different applied voltages, cubic non-linearity and non-linear damping, have been analytically studied both by means of the harmonic balance method and numerically. Deformations of two plates with a constraint have been omitted while modeling the micro-cantilever device as a system of one degree of freedom. Linear and non-linear tuning of parametrically excited designed and fabricated MEMS oscillators as well as chaos of the MEMS oscillators have been studied in Refs. [33,34]. In both cases, simple non-linear oscillators governed by non-autonomous secondorder ODEs with either constant or time periodic coefficients are used.

Owing to the overview of the state-of-the-art, let us emphasize now the importance of a single-mode approximation while studying the behavior of microbeams/microplates in MEMS devices and beyond. A direct use of FEM (Finite Element Method) or FDM (Finite Different Method) is expensive and time consuming. These sophisticated approaches are applied to study performance of already fabricated devices, rather than to design feedback control laws while manufacturing a MEMS device. The engineers expect simple rules and models for designing a MEMS system, which is contrary to the FEM/FDM approaches mostly using numerous variables to describe both the dynamical state of the investigated systems and complexity of the design space.

Therefore, the so-called reduced-order models or macromodels [35] are highly required to capture the most important characteristics of MEMS devices or other continuous mechanical systems employing only a few variables governed by linear/non-linear ODEs. However, on the contrary to purely engineering approaches, we offer a novel technique based on the FSDT and CPT matched with the variational Ritz method and the R-functions theory. As a result, the reduced-order models are yielded by advanced mathematical modeling. Presented models are efficient, accurate and allow to get accurate results relatively fast.

The paper is organized in the following way. The problem statement is defined in Section 2, including the governing differential equations. The method of solution is described in Section 3, whereas numerical examples are presented in Section 4 consisting of the validation study and the estimation of the instability zones of the studied plates. Concluding remarks (Section 5) finish our investigations reported in the paper. 


\section{Problem statement}

Let us consider a laminated plate, a structure of which is symmetrical with respect to its middle surface, consisting of $n$ layers of constant thickness. Assume that the plate is subjected to a periodic in-plane load of the form $p=p_{0}+p_{t} \cos \theta t$, where $p_{0}$ is a static component, $p_{t}$ is the amplitude of a periodic part, and $\theta$ is the frequency of an applied load. Note that all external forces are varied proportionally to the introduced control parameter $\lambda$. The smallest value of the parameter $\lambda$ is referred to as a critical value. The parametric excitation of the plate under periodic load is investigated by both classical thin plate theory and the first-order shear deformation theory (FSDT) [20], taking into account shear deformation. The classical theory yields the following differential equations governing dynamics of the laminated composite plates with parametric excitations:

$$
\begin{aligned}
& N_{11, x}+N_{12, y}=m_{1} u, t t, \\
& N_{12, x}+N_{22, y}=m_{1} v_{, t t}, \\
& M_{11, x x}+2 M_{12, x y}+M_{22 x, y y}+\left(N_{11} w_{, x x}+2 N_{12} w_{, x y}+N_{22} w_{, y y}\right) \\
& \quad=m_{1} w, t t+\epsilon m_{1} w_{, t} .
\end{aligned}
$$

Here $u, v$ and $w$ are the displacements of the plate in directions $O x, O y$ and $O z$, respectively, $\varepsilon_{d}$ is the damping coefficient, $N_{i j}$ and $M_{i j} M_{i j}(i, j=1,2)$ are the components of vectors stress resultants $N$ and moments $M$, respectively. It should be mentioned that a direct application of the first-order theory yields equations of complex forms due to an occurrence of the functions $\psi_{x}$ and $\psi_{y}$ describing the rotations perpendicular to the midsurface about the $y$ - and $x$ axes, respectively. In this case, the governing system of PDEs consists of the following five equations

$$
\begin{aligned}
& N_{11, x}+N_{12, y}=m_{1} u, t t, \\
& N_{12, x}+N_{22, y}=m_{1} v, t t, \\
& M_{11, x}+M_{12, y}-Q_{x}=m_{2} \psi_{x}, t t, \\
& M_{12, x}+M_{22, y}-Q_{y}=m_{2} \psi_{y, t t} \\
& Q_{x, x}+Q_{y, y}+\left(N_{11} w_{, x x}+2 N_{12} w_{, x y}+N_{22} w_{, y y}\right)=m_{1} w_{, t t}+\epsilon_{d} m_{1} w_{, t} .
\end{aligned}
$$

Let us present resultant forces $N=\left\{N_{11}, N_{22}, N_{12}\right\}$ and moments $M=\left\{M_{11}, M_{22}, M_{12}\right\}$ in the following matrix forms

$N=[C] \epsilon, M=[D] \chi$

where $\varepsilon=\left\{\varepsilon_{11}, \varepsilon_{22}, \varepsilon_{12}\right\}^{T}$ and $\chi=\left\{\chi_{11}, \chi_{22}, \chi_{12}\right\}^{T}$ are strains of the middle surface with the following components

$$
\begin{aligned}
\varepsilon_{11} & =u,_{x}+\frac{1}{2} w_{, x}^{2}, \quad \varepsilon_{22}=v, y+\frac{1}{2} w_{, y}^{2}, \quad \varepsilon_{12}=u, y+v_{, x}+w_{, x} w_{, y}, \\
\chi_{11} & =\delta \psi_{x}, x-(1-\delta) w_{, x x}, \quad \chi_{22}=\delta \psi_{y, y}-(1-\delta) w_{, y y}, \\
\chi_{12} & =\delta\left(\psi_{x}, y+\psi_{y}, x\right)-2(1-\delta) w_{, x y} .
\end{aligned}
$$

The introduced multiplier $\delta$ plays the role of a tracing constant which takes the values 1 and 0 for the FSDT and CPT, respectively. As usual, the subscripts following a comma stand for partial differentiation.

The matrices $[C]$ and $[D]$ present in formulas (9) have the following explicit forms

$$
[C]=\left[\begin{array}{lll}
C_{11} & C_{12} & C_{16} \\
C_{12} & C_{22} & C_{26} \\
C_{16} & C_{26} & C_{66}
\end{array}\right],[D]=\left[\begin{array}{ccc}
D_{11} & D_{12} & D_{16} \\
D_{12} & D_{22} & D_{26} \\
D_{16} & D_{26} & D_{66}
\end{array}\right]
$$

and the constants $C_{i j}$ and $D_{i j}$ are related to reduced stiffness coefficients of the plate and are defined by the following formulas

$$
\begin{gathered}
\left(C_{i j}(x, y), D_{i j}(x, y)\right)=\sum_{m=1}^{M} \int_{h_{m}(x, y)}^{h_{m+1}(x, y)} B_{i j}^{(m)}\left(1, z^{2}\right) d z, \quad(i, j=1,2,6 .) \\
C_{i j}(x, y)=K_{i} \sum_{m=1}^{M} \int_{h_{m}(x, y)}^{h_{m+1}(x, y)} B_{i j}^{(m)} d z,(i, j=4,5),\left(m_{1}, m_{2}\right) \\
=\sum_{m=1}^{n} \int_{h_{m}}^{h_{m+1}}(1, z) \rho^{(m)} d z
\end{gathered}
$$

where $B_{i j}^{(m)}$ describe a mechanical characteristics and $\rho^{(m)}$ is the material density of the $m$-th layer.

In the present study the shift factors $K_{i}(i=4,5)$ are taken equal to 5/6. Transverse forces $Q_{x}, \quad Q_{y}$ are defined in the following way

$\{Q\}=\left\{\begin{array}{l}Q_{x} \\ Q_{y}\end{array}\right\}=\left[\begin{array}{ll}C_{55} C_{54} \\ C_{45} C_{44}\end{array}\right]\left\{\begin{array}{l}\varepsilon_{13} \\ \varepsilon_{23}\end{array}\right\}$

where

$\epsilon_{13}=\delta\left(w_{, x}+\psi_{x}-\frac{u}{R_{x}}\right), \epsilon_{23}=\delta\left(w_{, y}+\psi_{y}-\frac{v}{R_{y}}\right)$.

The systems (1)-(3) and (4)-(8) of the motion equations are supplemented with the corresponding boundary and initial conditions.

\section{Method of solution}

In what follows we assume that the subcritical state may be inhomogeneous. Therefore, we need to determine the subcritical state first, and then to solve a linear vibration problem for the plate loaded on its midplane. The detailed description of the solution methods involved in this step in the framework of classical plate theory can be found in Refs. $[12,13]$. Let us present an algorithm of the solution for the case of the FSTD. For convenience, the strain and stress resultants are presented as sums of linear and non-linear parts in the following way

$\epsilon=\epsilon^{(L)}+\epsilon^{(N)}, N=N^{(L)}+N^{(N)}$

where

$\epsilon^{(L)}=\left(\epsilon_{11}^{(L)}, \epsilon_{22}^{(L)}, \epsilon_{12}^{(L)}\right)^{T}, \epsilon^{(N)}=\left(\epsilon_{11}^{(N)}, \epsilon_{22}^{(N)}, \epsilon_{12}^{(N)}\right)^{T}$

$\epsilon_{11}^{(L)}=u_{, x}, \epsilon_{22}^{(L)}=v_{, y}, \epsilon_{12}^{(L)}=v_{, x}+u_{, y}$

$\epsilon_{11}^{(N)}=\frac{1}{2}\left(w_{, x}\right)^{2}, \epsilon_{22}^{(N)}=\frac{1}{2}(w, y)^{2}, \epsilon_{12}^{(N)}=w, x w, y$

Then, the stress resultants $N^{(L)}=\left(N_{11}^{(L)}, N_{22}^{(L)}, N_{12}^{(L)}\right)^{T}$ and $N^{(N)}=$ $\left(N_{11}^{(N)}, N_{22}^{(N)}, N_{12}^{(N)}\right)^{T}$ can be recast to the following form

$N^{(L)}=[C] \epsilon^{(L)}, N^{(N)}=[C] \epsilon^{(N)}$.

Suppose that all external forces are varied proportionally to the parameter $\lambda$. In order to determine the subcritical stress state of the plate, we have considered the system

$N_{11}^{(L)}, x+N_{12}^{(L)}, y=0$,

$N_{12}^{(L)}, x+N_{22}^{(L)}, y=0$,

supplemented with the following boundary conditions on the loaded edges

$N_{n}^{(L)}(u, v)=-1, T_{n}^{(L)}(u, v)=0$ 
The operators $N_{n}^{(L)}, T_{n}^{(L)}$ are defined by the following formulas

$N_{n}^{(L)}=N_{11}^{(L)} l^{2}+N_{22}^{(L)} m^{2}+2 N_{12}^{(L)} l m, T_{n}^{(L)}=N_{12}^{(L)}\left(l^{2}-m^{2}\right)+\left(N_{11}^{(L)}-N_{22}^{(L)}\right) l m$

where $l=\cos (\vec{n}, O x), m=\cos (\vec{n}, O y)$, and $\vec{n}$ is a vector normal to the border of the domain.

The boundary problem (18) and (19) is solved by the variational Ritz's method combined together with the R-functions theory (RFM). Observe that the variational formulation of the problem (18) and (19) is reduced to that of finding the minimum of the functional

$I(u, v)=\frac{1}{2} \iint_{\Omega}\left(N_{11}^{(L)} \epsilon_{11}^{(L)}+N_{22}^{(L)} \epsilon_{22}^{(L)}+N_{12}^{(L)} \epsilon_{12}^{(L)}\right) d \Omega+\int_{\partial \Omega_{1}} N_{n}^{(L)}(u l+v m) d \Omega_{1}$,

where $d \Omega_{1}$ is the loaded part of the border. We are aimed at finding the minimum of the functional on the set of basic functions constructed using the R-functions theory $[11,22]$.

Let us denote the solution of the system (18) by $u^{(1)}(x, y), v^{(1)}(x, y)$. The values of the stress resultants, corresponding to the value of the parameter $\lambda$ equal to 1 , are denoted by $N_{i j}^{\left(L_{0}\right)}$ $(i, j=1,2)$. Then, the stress resultants $N^{\left(L_{0}\right)}=\left(N_{11}^{\left(L_{0}\right)}, N_{22}^{\left(L_{0}\right)}, N_{12}^{\left(L_{0}\right)}\right)$ are defined by formula (17), where

$\epsilon^{(L)}=\left(\epsilon_{11}^{(L)}, \epsilon_{22}^{(L)}, \epsilon_{12}^{(L)}\right)^{T}, \epsilon_{11}^{(L)}=u^{(1)}{ }_{x}, \epsilon_{22}^{(L)}=v^{(1)}{ }_{, y}, \epsilon_{12}^{(L)}=v^{(1)}{ }_{x}+u^{(1)}{ }_{y}$

Since all external forces are varied proportionally to the parameter $\lambda$, the critical value of the parameter $\lambda$ may be found as the smallest eigenvalue of the following functional

$$
\begin{aligned}
I\left(w, \psi_{x}, \psi_{y}\right)= & \frac{1}{2} \iint_{\Omega}\left(M_{11} \chi_{11}+M_{22} \chi_{22}+M_{12} \chi_{12}+\delta\left(Q_{x} \epsilon_{13}+Q_{y} \epsilon_{23}\right)\right. \\
& \left.+\lambda\left(N_{11}^{\left(L_{0}\right)}(w, x)^{2}+N_{22}^{\left(L_{0}\right)}(w, y)^{2}+N_{12}^{\left(L_{0}\right)} w, x w, y\right)\right) d \Omega .
\end{aligned}
$$

The classical problem regarding linear vibrations of the compressed plate is also reduced to a counterpart variational problem yielding stationary value of the Lagrange's functional:

$$
\begin{aligned}
I\left(w, \psi_{x}, \psi_{y}\right)= & \frac{1}{2} \iint_{\Omega}\left(M_{11} \chi_{11}+M_{22} \chi_{22}+M_{12} \chi_{12}+\delta\left(Q_{x} \epsilon_{13}+Q_{y} \epsilon_{23}\right)\right. \\
& \left.+p_{0}\left(N_{11}^{\left(L_{0}\right)}(w, x)^{2}+N_{22}^{\left(L_{0}\right)}\left(w_{, y}\right)^{2}+N_{12}^{\left(L_{0}\right)} w, x w, y\right)\right) d \Omega \\
& -\frac{\Omega_{L}^{2}}{2} \iint_{\Omega} \rho\left(h w_{0}^{2}+\delta \frac{h^{3}}{12}\left(\psi_{x}^{2}+\psi_{y}^{2}\right)\right) d \Omega
\end{aligned}
$$

Note that while solving the linear problem, all inertia forces, including membrane and rotary ones, are taken into account and the critical load value may be found together with eigenvalues (dynamic approach). Indeed, as it follows from (24), each value of the parameter $p_{0}$ is associated with a set of eigenvalues related to different compressed loads. The plate vibrates about its equilibrium position until all its frequencies are real, i.e. its equilibrium configuration becomes stable. However, we can obtain zero or imaginary value of the natural frequency by means of increasing the parameter $p_{0}$. In this case, the equilibrium position would be unstable. The parameter value $p_{0}$, corresponding to the smallest non-negative value of the natural frequency, is referred to as the critical value. Now, the estimated eigenfunctions $w_{i}^{(c)}(x, y), u_{i}^{(c)}(x, y)$, $v_{i}^{(c)}(x, y), \psi_{x i}^{(c)}(x, y), \psi_{y i}^{(c)}(x, y)$ of the linear vibration problem, corresponding to the load $p_{0}$, are used to present the solution of the non-linear problem (1)-(3) or (4)-(8) in the form of the following truncated series:

$w(x, y, t)=\sum_{i=1}^{n} y_{i}(t) w_{i}^{(c)}(x, y)$

$\psi_{x}(x, y, t)=\delta \sum_{i=1}^{n} y_{i}(t) \psi_{x i}^{(c)}(x, y), \psi_{y}(x, y, t)=\delta \sum_{i=1}^{n} y_{i}(t) \psi_{y i}^{(c)}(x, y)$,

$u(x, y, t)=u_{1}(x, y) p+\sum_{i=1}^{n} \sum_{j=1}^{n} y_{i} y_{j} u_{i j}, v(x, y, t)=v_{1}(x, y) p+\sum_{i=1}^{n} \sum_{j=1}^{n} y_{i} y_{j} v_{i j}$,

where $y_{k}(t)$ are unknown functions to be defined further. Note that the functions $u_{i j}, v_{i j}$ should satisfy Eqs. (1) and (2), which we present in the form of displacements

$L_{11}\left(u_{i j}\right)+L_{12}\left(v_{i j}\right)=-N l_{1}^{(2)}\left(w_{i}, w_{j}\right)$,

$L_{21}\left(u_{i j}\right)+L_{22}\left(v_{i j}\right)=-N l_{2}^{(2)}\left(w_{i}, w_{j}\right)$.

The linear operators $L_{11}, L_{22}, L_{12}, L_{21}$ are defined as follows

$L_{11}=C_{11}()_{, x x}+2 C_{16}()_{, x y}+C_{66}()_{, y y}, L_{22}=C_{66}()_{, x x}+2 C_{26}()_{, x y}+C_{22}(), y y$,

$L_{12}=L_{21}=C_{16}()_{, x x}+\left(C_{12}+C_{66}\right)()_{, x y}+C_{26}(), y y$,

whereas the non-linear operators $N l_{1}^{(2)}\left(w_{i}^{(c)}, w_{j}^{(c)}\right), N l_{2}^{(2)}\left(w_{i}^{(c)}, w_{j}^{(c)}\right)$ follow

$N l_{1}^{(2)}\left(w_{i}^{(c)}, w_{j}^{(c)}\right)=w_{i, x} L_{11} w_{j}+w_{i, y} L_{12} w_{j}$,

$N l_{2}^{(2)}\left(w_{i}^{(c)}, w_{j}^{(c)}\right)=w_{i, x} L_{12} w_{j}+w_{i, y} L_{22} w_{j}$

The system of Eq. (28) supplemented with the corresponding boundary conditions is solved by means of RFM. Substituting formulas (26), (27) for functions $u, v, w, \psi_{x}, \psi_{y}$ into equations of motion (1) and (2) or (4) and (5), ignoring inertia terms and applying the Bubnov-Galerkin procedure, we obtain the following non-linear system of ODEs regarding the unknown functions $y_{m}(t)$ :

$$
\begin{gathered}
y_{m}^{\prime \prime}(t)+\epsilon_{d} y_{m}^{\prime}(t)+\Omega_{m}^{2}\left(y_{m}(t)+p_{t} \cos \theta t \sum_{k=1}^{n} \alpha_{k}^{(m)} y_{k}(t)\right. \\
\left.+\sum_{i=1}^{n} \sum_{j=1}^{n} \sum_{k=1}^{n} \gamma_{i j k}^{(m)} y_{i}(t) y_{j}(t) y_{k}(t)\right)=0
\end{gathered}
$$

where $m=1, \ldots, n$, and the coefficients are defined as follows

$\alpha_{k}^{(m)}=-\frac{\iint_{\Omega} N l_{31}\left(u_{1}, v_{1}, w_{k}\right) w_{m} d \Omega}{m_{1} \Omega_{m}^{2}\left\|w_{m}\right\|^{2}}, \quad \gamma_{i j k}^{(m)}=-\frac{\iint_{\Omega} N l_{32}\left(u_{i j}, v_{i j}, w_{k}\right) w_{m} d \Omega}{m_{1} \Omega_{m}^{2}\left\|w_{m}\right\|^{2},}$

where

$N l_{31}\left(u_{1}, v_{1}, w_{k}\right)=N_{11}^{(L)} w_{k}, x x+N_{22}^{(L)} w_{k}, y y+2 N_{12}^{(L)} w_{k}, x y$
$N l_{32}\left(u_{i j}, v_{i j}, w_{k}\right)=N_{11} w_{k}, x x+N_{22} w_{k}, y y+2 N_{12} w_{k}, x y$.

It is known that the most important instability region is the primary one with the period $2 \mathrm{~T}$ (see, for instance, monograph [36]). It is usually much larger than a secondary instability region corresponding to the period T. Furthermore, the results needed for practical engineering-oriented applications can be obtained taking into account only the first-order approximation of the major instability boundaries [4,5]. This is why only the first-order approximation will be used in the present study. Let us consider in detail one-mode approximation governed by the following 
equations:

$w(x, y, t)=y(t) w_{1}(x, y), \psi_{x}(x, y, t)=\delta y(t) \psi_{x 1}^{(c)}(x, y)$,

$\psi_{y}(x, y, t)=\delta y(t) \psi_{y 1}^{(c)}(x, y)$,

$u(x, y, t)=p(t) u_{1}(x, y)+y^{2}(t) \cdot u_{11}(x, y)$

$v(x, y, t)=p(t) v_{1}(x, y)+y^{2}(t) \cdot v_{11}(x, y)$.

In this case, the system of Eq. (32) is reduced to only one equation with periodic coefficients of the following form

$y^{\prime \prime}(t)+\varepsilon y^{\prime}(t)+\left(1+\alpha p_{t} \cos \theta t+\gamma y^{2}(t)\right) y(t)=0$

where

$y(t)=y_{1}(t), \Omega_{L}=\Omega_{1}, \alpha=\alpha_{1}^{(1)}, \gamma=\gamma_{111}^{(1)}$.

In order to determine the regions of dynamic instability with Bolotin's method [4], Eq. (36) is transformed to the following, more convenient form

$y^{\prime \prime}(t)+2 \varepsilon_{1 d} y_{m}^{\prime}(t)+\Omega_{L}^{2}(1-2 k \cos \theta t) y(t)+\gamma y^{3}(t)=0$

where

$2 k=-\alpha \cdot p_{t}, \varepsilon_{1 d}=\varepsilon_{d} / 2$

Furthermore, we neglect the non-linear term while determining instability regions $[4](\gamma=0)$. The obtained linear equation is well-studied. As it has been shown by Bolotin [4], the major instability region is situated near $\theta=2 \Omega_{L}$ and bounded by the following curve

$2 \Omega_{L} \sqrt{1-} \sqrt{k^{2}}-\left(\frac{\Delta}{\pi}\right)^{2} \leq \theta \leq 2 \Omega_{L} \sqrt{1+} \sqrt{k^{2}}-\left(\frac{\Delta}{\pi}\right)^{2}$

where $\Delta=\frac{2 \pi \varepsilon_{1}}{\Omega_{1}}$. The relation between the frequency ratio and the amplitude of non-linear vibrations after a loss of stability, according to [4], has the following form

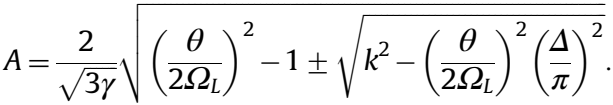

\section{Numerical results}

\subsection{Validation study}

Since the investigation of laminated plate stability problems by means of joint application of the RFM and the FSDT is considered for the first time, we are aimed at showing the accuracy of the proposed approach by comparing our results with the results from the literature, using examples of several tasks.

Task 1. Let us consider the third and the fourth cross-ply square laminated plates (see Fig. 1) subjected to uni-axial load (i.e. $N_{11}=-p$ ). The layers have the same thickness. Orthotropic layers with the following engineering constants are used:

$E_{1} / E_{2}=40, G_{12}=G_{13}=0.6 E_{2}, G_{23}=0.5 E_{2}, \nu_{12}=0.25$.

The following 'simply-supported' boundary conditions are assumed:

$u=w=\psi_{x}=M_{22}=N_{22}=0, y= \pm \frac{b}{2}$

$v=w=\psi_{y}=M_{11}=0, \quad N_{11}=-p, \quad x= \pm \frac{a}{2}$.

Note that in the studied case the stress distribution is uniform in the $x y$ plane. However, in order to check our developed software, we have solved the problem by applying the general algorithm described above. The obtained numerical results for nondimensional critical loads of cross-ply laminated plates and for

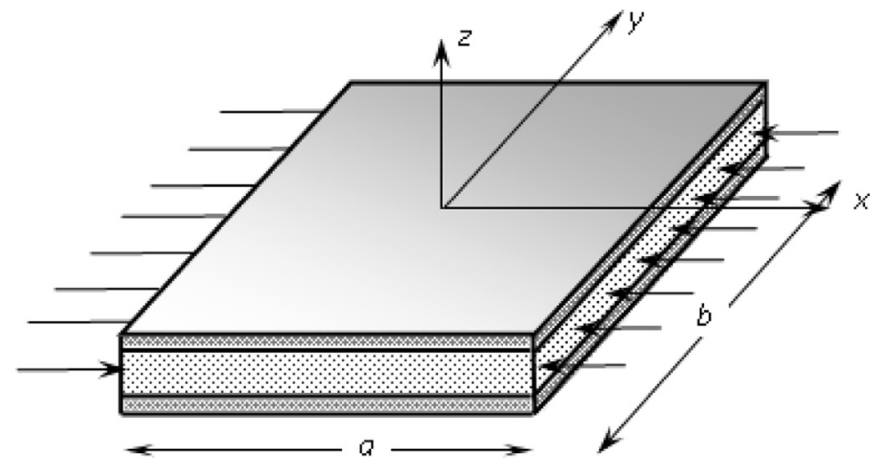

Fig. 1. A plate and the loading way.

Table 1

Buckling coefficient $p_{c r}=N_{11}(2 b)^{2} / E_{2} h^{3}$ for different values of thickness of crossply square plates, compared with the results presented in Ref. [21].

\begin{tabular}{|c|c|c|c|c|c|c|}
\hline \multirow[t]{2}{*}{$a / h$} & \multicolumn{3}{|c|}{$\left(0^{\circ} / 90^{\circ} / 0^{\circ}\right)$} & \multicolumn{3}{|c|}{$\left(0^{\circ} / 90^{\circ} / 90^{\circ} / 0^{\circ}\right)$} \\
\hline & $\begin{array}{l}\text { RFM } \\
\text { (FSDT) }\end{array}$ & $\begin{array}{l}{[21],} \\
\text { FSDT }\end{array}$ & $\begin{array}{l}{[21],} \\
\text { HSDT }\end{array}$ & $\begin{array}{l}\text { RFM } \\
\text { (FSDT) }\end{array}$ & $\begin{array}{l}{[21],} \\
\text { FSDT }\end{array}$ & $\begin{array}{l}{[21],} \\
\text { HSDT }\end{array}$ \\
\hline 10 & 21.81 & 21.64 & 22.16 & 23.47 & 23.27 & 23.85 \\
\hline 12.5 & 25.50 & 25.14 & 25.59 & 26.84 & 26.52 & 27.03 \\
\hline 20 & 31.41 & 30.66 & 30.92 & 32.41 & 31.43 & 31.74 \\
\hline 25 & 33.23 & 32.33 & 32.51 & 34.01 & 32.87 & 33.09 \\
\hline 50 & 35.60 & 34.88 & 34.94 & 36.50 & 35.04 & 35.10 \\
\hline 100 & 36.50 & 35.59 & 35.60 & 37.10 & 35.63 & 35.64 \\
\hline
\end{tabular}

different values of the plate thickness are compared with the corresponding values reported in Table 1.

By looking at the results presented in Table 1 one can notice that the maximum deviation between the results does not exceed $4 \%$.

Task 2. Let us consider a static stability for a three- and fivelayer cross-ply plate with a symmetrical structure and boundary conditions of two types:

a) Boundary conditions of the 1st type (see Fig. 2(a))

$N_{11}=0, v=w=M_{11}=\psi_{y}=0, x= \pm \frac{a}{2}$

$N_{22}=-p, \quad u=w=M_{22}=\psi_{x}=0, y= \pm \frac{b}{2}$

b) Boundary condition of the 2nd type (see Fig. 2(b))

$N_{22}=-p, u=w=M_{22}=\psi_{x}=0, y=\frac{b}{2}$

$N_{22}=0, u=v=w=\psi_{x}=\psi_{y}=0, y=-\frac{b}{2}$

$N_{11}=0, v=w=M_{11}=\psi_{y}=0, x= \pm \frac{a}{2}$

Characteristics of the material and geometrical parameters are taken as follows [18]:

$G_{12} / E_{2}=0.6, G_{13} / E_{2}=0.5, G_{23} / E_{2}=0.5, \nu_{12}=0.25, b / a=1, h / a=0.1$

The values of the loading critical parameter $p_{c r}=\frac{N_{22} a^{2}}{E_{2} h^{3}}$ for various values $E_{1} / E_{2}$ are presented in Table 2.

It can be seen that for the first-type boundary conditions the results obtained by the RFM are close to those reported in Ref. [18]. We also note that the values of critical loading parameters for plates loaded along one side and two opposite sides are practically the same. This can be explained by the effect of balancing the load by the boundary conditions. Table 2 confirms that application of the CPT to plates of moderate thickness leads to an obvious error. 

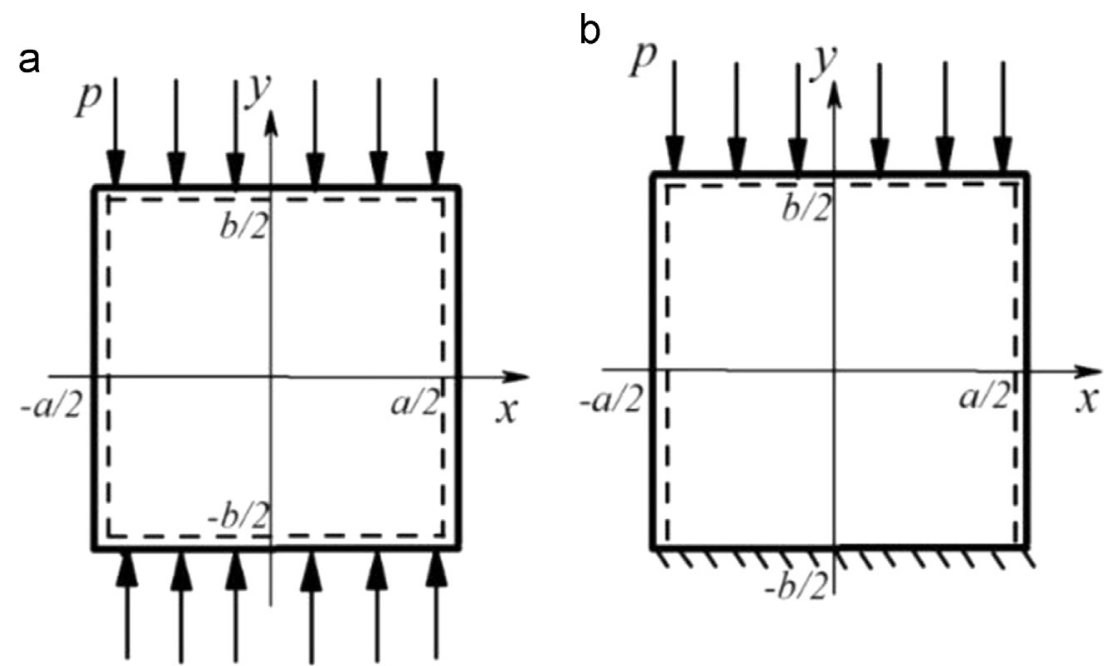

Fig. 2. The plate midsurface, ways of applying the load and types of boundary conditions.

Table 2

Values of critical load parameter $p_{c r}=N_{22} a^{2} /\left(E_{2} h^{3}\right), h / a=0.1$.

\begin{tabular}{|c|c|c|c|c|c|c|c|c|}
\hline \multirow[t]{2}{*}{ B.C } & \multirow[t]{2}{*}{ Theory } & \multirow[t]{2}{*}{ Method } & \multirow{2}{*}{$\begin{array}{l}\text { No of } \\
\text { layers }\end{array}$} & \multicolumn{5}{|l|}{$E_{1} / E_{2}$} \\
\hline & & & & 3 & 10 & 20 & 30 & 40 \\
\hline $1-\mathrm{st}$ & CPT & RFM & $30^{\circ} / 90^{\circ} / 0^{\circ}$ & 5.75 & 11.49 & 19.72 & 27.95 & 36.17 \\
\hline $1-\mathrm{st}$ & CPT & [18] & & 5.75 & 11.49 & 19.71 & 27.94 & 36.16 \\
\hline 1 -st & FSDT & RFM & & 5.40 & 9.87 & 14.98 & 19.02 & 22.31 \\
\hline 1 -st & FSDT & [18] & & 5.40 & 9.96 & 15.35 & 19.76 & 23.45 \\
\hline 2-nd & FSDT & RFM & & 5.36 & 9.86 & 14.98 & 19.02 & 22.31 \\
\hline 1 -st & $\mathrm{CPT}$ & RFM & $50^{\circ} / 90^{\circ} / 0^{\circ} /$ & 5.757 & 11.499 & 19.726 & 27.954 & 36.183 \\
\hline $1-\mathrm{st}$ & $\mathrm{CPT}$ & [18] & $90^{\circ} / 0^{\circ}$ & 5.754 & 11.492 & 19.712 & 27.936 & 36.160 \\
\hline 1 -st & FSDT & RFM & & 5.41 & 10.08 & 15.74 & 20.49 & 24.54 \\
\hline $1-s t$ & FSDT & [18] & & 5.41 & 10.14 & 15.96 & 20.91 & 25.19 \\
\hline 2-nd & FSDT & RFM & & 5.37 & 10.06 & 15.73 & 20.48 & 24.54 \\
\hline
\end{tabular}

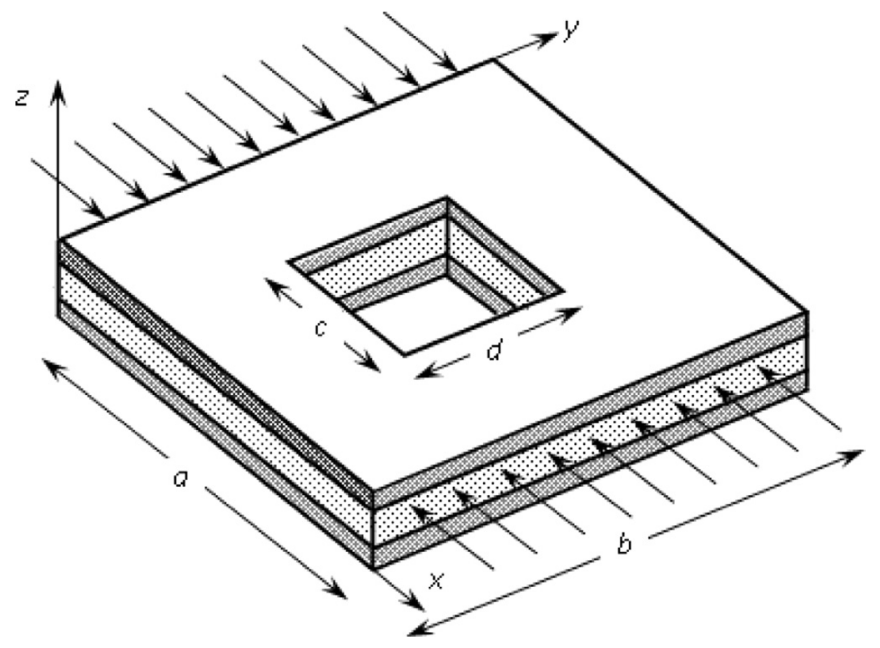

Fig. 3. The shape of a laminated plate.

Analyzing the influence of the degree of orthotropy given by the ratio of Young's modulus $E_{1} / E_{2}$ (Table 2), one can see that an increase in $E_{1} / E_{2}$ yields to an increase in the critical load parameter for both types of boundary conditions. In addition, a disagreement between the results obtained by CPT and FSDT significantly increases if the ratio $E_{1} / E_{2}$ increases.

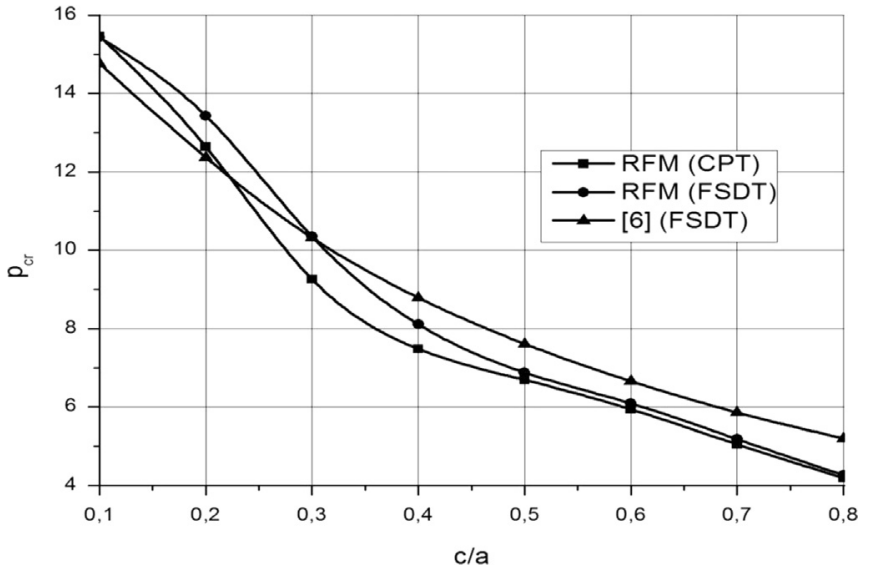

Fig. 4. A comparison of the critical load with known results [6].

Task 3. Let us investigate a rectangular laminated plate with a central square cutout (Fig. 3) and the following material properties [6]:

$E_{1}=141 \mathrm{GPa}, E_{2}=9.23 \mathrm{GPa}, G_{12}=G_{13}=5.95 \mathrm{GPa}$,

$G_{23}=2.96 \mathrm{GPa}, \nu_{12}=0.313$.

Suppose that the plate is simply-supported on its outer contour, free on its inner contour, and has a symmetric structure with cross-ply $\left[0^{\circ} / 90^{\circ}\right]_{s}$. The used geometrical parameters are: $a=b=0.5 \mathrm{~m}, h=0.005 \mathrm{~m}$. Since the stress field is non-uniform due to the cutout, the plane stress analysis is needed. Therefore, in this case, we should apply all steps of the developed algorithm. Fig. 4 presents a comparison of the obtained results to the known ones [6] for different sizes of the cutout.

It follows from the performed comparison that the divergence of the results presented in Fig. 3 depends on the cutout size and increases with an increase in the cutout size, beginning with the ratio $c / a=0.4$. If the ratio $c / a$ satisfies inequality $0.1 \leq c / a<0.4$, then the difference between compared results does not exceed $5 \%$. We think that it can be connected with the used systems of approximation of indefinite components (system of power polynomials). Obviously, in this case, the application of splines is preferable. 

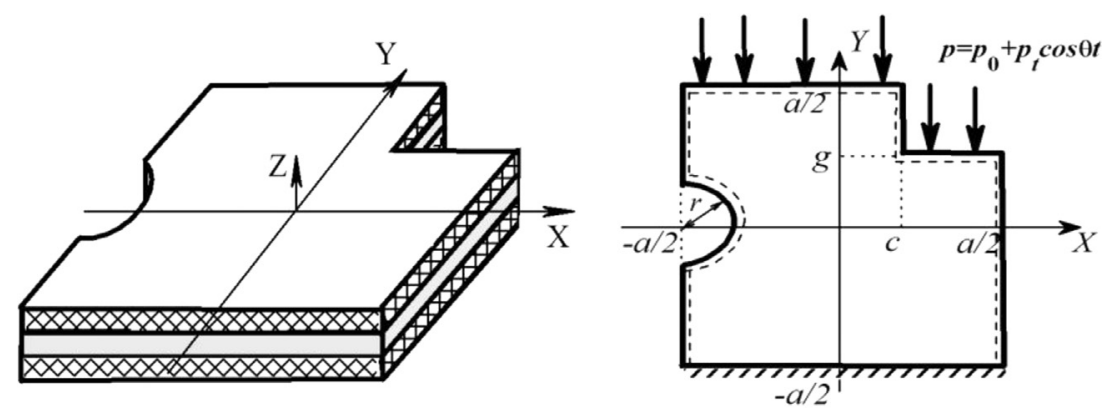

Fig. 5. The geometric form of a plate.

4.2. Dynamic instability and parametric vibrations of the plate with external cutouts of different forms

It is evident that, in practice, many structural members are supported only on one side and may be loaded on their remaining parts. In order to demonstrate the capabilities of the proposed approach, we have considered laminated plate with two cutouts of different forms, provided that the plate is clamped on one side, compressed on the opposite side and simply supported on the remaining parts.

In other words, we deal with mixed boundary conditions. Application of the FSDT requires constructing a new solution structure satisfying the given boundary condition. Below we present a solution to the formulated problem.

Task 1. Let us investigate a three-layer plate with two external cutouts: the round and rectangular (see Fig. 5) ones. The plate has a symmetrical structure and constant thickness $h$. The thickness of all layers is the same.

Below the considered boundary conditions are presented for the classical and the first-order deformation theories.

$$
\begin{aligned}
& \text { a) CPT } \\
& N_{22}=-p, N_{12}=0, w=0, M_{22}=0, \forall(x, y) \in \partial \Omega_{1}, \\
& u=0, v=0, w=0, \frac{\partial w}{\partial n}=0, y=-\frac{a}{2},(x, y) \in \partial \Omega_{2}, \\
& N_{n}=0, T_{n}=0, w=0, M_{n}=0,(x, y) \in \partial \Omega_{3}=\partial \Omega /\left(\partial \Omega_{1} \cup \partial \Omega_{2}\right) .
\end{aligned}
$$

\section{b) FSDT}

$N_{22}=-p, N_{12}=0 \forall(x, y) \in \partial \Omega_{1}$

$u=v=w=\psi_{x}=\psi_{y}=0, \forall(x, y) \in \partial \Omega_{2}$

$N_{n}=T_{n}=0, w=0, M_{n}=0, \quad \forall(x, y) \in \partial \Omega_{3}=\partial \Omega /\left(\partial \Omega_{1} \cup \partial \Omega_{2}\right)$

$\psi_{y n}=0, \quad \forall(x, y) \in \partial \Omega$

where

$\psi_{y n}=-\psi_{x} m+\psi_{y} l, \quad l=\cos (n, O x), \quad m=\cos (n, O y)$

$\partial \Omega_{1}=\left\{(x, y) \mid\left(y=\frac{a}{2},-\frac{a}{2} \leq x \leq c\right) \cup\left(y=g, c \leq x \leq \frac{a}{2}\right)\right\}$

$\partial \Omega_{2}=\left\{(x, y) \mid\left(y=-\frac{a}{2},-\frac{a}{2} \leq x \leq \frac{a}{2}\right)\right\}$

Due to the fact that the considered plate has cutouts, mixed boundary conditions, and it is loaded along one edge, it exhibits an inhomogeneous subcritical state. In this case, solution of the problem is complicated and all stages of our algorithm have to be performed.

Since the R-functions theory is applied, we should construct the solution structure. In order to satisfy the main boundary conditions, the following structures of solutions are proposed for CPT and FSDT, respectively:

a) Structures of solution for CPT:

$$
u=\omega_{1} \Phi_{1}, v=\omega_{1} \Phi_{2}, w=\omega \omega_{1} \Phi_{3} .
$$

Here $\omega_{1}=0$ refers to the equation of a clamped part $\left(\partial \Omega_{2}\right)$. Function $\omega_{1}$ takes the form of $\omega_{1}=f_{0}$, where $f_{0}=\frac{a}{2}+y \geq 0$. To implement the structures of solution (50), we have to form a normalized equation [22] of the boundary domain $\omega(x, y)=0$ :

$\omega=f_{1} \wedge_{0} f_{2} \wedge_{0} f_{3} \wedge_{0}\left(f_{4} \vee_{0} f_{5}\right)$,

where

$f_{1}=\frac{1}{a}\left(\left(\frac{a}{2}\right)^{2}-x^{2}\right) \geq 0, f_{2}=\frac{1}{a}\left(\left(\frac{a}{2}\right)^{2}-y^{2}\right) \geq 0$,

$f_{3}=\frac{1}{2 r}\left(\left(x+\frac{a}{2}\right)^{2}+y^{2}-r^{2}\right) \geq 0$,

$f_{4}=g-y \geq 0, f_{5}=c-x \geq 0$.

Symbols $\wedge_{0}, \vee_{0}$ in (37) denote R-operations [11,22], presented in the following form

$x \wedge{ }_{0} y=x+y-\sqrt{x^{2}+y^{2}}, x \vee{ }_{0} y=x+y+\sqrt{x^{2}+y^{2}}$.

b) Structures of solution for FSDT:

$u=\omega_{1} \Phi_{1}, v=\omega_{1} \Phi_{2}, w=\omega \Phi_{3}$,

$\psi_{x}=\omega,{ }_{x} \Phi_{4}+\omega \Phi_{5}, \psi_{y}=\left(\omega, y \Phi_{4}+\omega \Phi_{6}\right) \omega_{1}$

Obviously, all main (geometric) boundary conditions are satisfied for any of indefinite components $\Phi_{i},(i=1, \ldots, 6)$. In order to check the boundary condition (48), let us note that the equation of the boundary condition is normalized. Hence, directional cosines $l=\cos (n, O x), m=\cos (n, O y)$ may be continued to the inside of the domain with the help of the following formulas

$l=\cos \alpha=-\omega_{, x}, m=\sin \alpha=-\omega_{, y}$

Substituting (55) and (56) into (49), we can confirm that the boundary condition (48) is satisfied. In order to construct the basic functions, indefinite components $\Phi_{i}, i=1, \ldots, 6$, should be considered in formulas (50), (54), and (55), in the form of the following truncated series

$\Phi_{i}=\sum_{k=1}^{k=N_{i}} a_{k}^{(i)} \phi_{k}^{(i)}$,

where $\left\{\phi_{k}^{(i)}\right\}$ are the known complete systems of functions (for instance, power or Chebyshev's polynomials, trigonometric functions, splines, or others). In the present study, power polynomials have been used in Eq. (57) to get numerical results. Substituting 
(57) into Eqs. (50) or (54) and (55) yields

$u=\sum_{i=1}^{R_{1}} a_{i} u_{i}, v=\sum_{i=1}^{R_{2}} a_{i} v_{i}, w=\sum_{i=1}^{R_{3}} a_{i} w_{i}$,

$\psi_{x}=\sum_{i=R_{3}+1}^{R_{4}} a_{i} \omega,{ }_{x} \psi_{i}+\sum_{i=R_{4}+1}^{R_{5}} a_{i} \psi_{x i}, \psi_{y}=\sum_{i=R_{3}+1}^{R_{4}} a_{i} \omega, y \psi_{i}+\sum_{i=R_{5}+1}^{R_{6}} a_{i} \psi_{y i}$,

where $u_{i}=\omega \varphi_{i}^{(1)}, v_{i}=\omega \varphi_{i}^{(2)}, w_{i}=\omega \varphi_{i}^{(3)}, \psi_{i}=\varphi_{i}^{(4)}, \psi_{x i}=\omega \varphi_{i}^{(5)}$, $\psi_{y i}=\omega \varphi_{i}^{(6)}$ are the admissible functions that satisfy boundary conditions. The unknown coefficients $a_{i}, i=\sum_{j=1}^{6} R_{j}$ are calculated by finding a stationary point of the corresponding functional. In order to determine the needed numbers of the coordinate functions, we have studied convergence of frequency parameters of the first vibration mode $\Lambda_{1}=\frac{\lambda_{1}(2 a)^{2}}{h} \sqrt{\frac{12\left(1-\nu_{12} \nu_{21}\right) \rho}{E_{2}}}$ for cross-ply three-

Table 3

Convergence of frequency parameters $\Lambda_{i}=\frac{\lambda_{i} a^{2}}{h} \sqrt{\frac{12\left(1-\nu_{12} \nu_{21}\right) \rho}{E_{2}}}$ of a cross-ply laminated plate $\left(90^{\circ} / 0^{\circ} / 90^{\circ}\right)$, obtained with the CPT (Material I (60), $\frac{h}{a}=0.01$ ).

\begin{tabular}{cllll}
\hline \multicolumn{1}{l}{$R_{3}$} & $\Lambda_{1}$ & $\Lambda_{2}$ & $\Lambda_{3}$ & $\Lambda_{4}$ \\
\hline $55(9)$ & 70.49 & 116.97 & 162.76 & 170.30 \\
$66(10)$ & 70.24 & 116.49 & 162.39 & 169.90 \\
$78(11)$ & 69.98 & 116.03 & 162.15 & 169.67 \\
$91(12)$ & 69.72 & 115.66 & 161.83 & 169.43 \\
$105(13)$ & 69.56 & 115.17 & 161.52 & 169.15 \\
\hline
\end{tabular}

\section{Table 4}

Convergence of frequency parameters $\Lambda_{i}=\frac{\lambda_{i} a^{2}}{h} \sqrt{\frac{12\left(1-\nu_{12} \nu_{21}\right) \rho}{E_{2}}}$ of a cross-ply laminated plate $\left(90^{\circ} / 0^{\circ} / 90^{\circ}\right)$, obtained with the FSTD (Material I, (60), $\frac{h}{a}=0.01$ ).

\begin{tabular}{llllll}
\hline$R_{3}$ & $R_{5} / R_{6} / R_{4}$ & $\Lambda_{1}$ & $\Lambda_{2}$ & \multicolumn{1}{l}{$\Lambda_{3}$} & \multicolumn{1}{c}{$\Lambda_{4}$} \\
\hline \multirow{2}{*}{55} & $45 / 45 / 55$ & 72.85 & 120.39 & 168.22 & 174.51 \\
& $45 / 45 / 66$ & 72.76 & 120.04 & 167.91 & 174.03 \\
& $55 / 55 / 55$ & 72.61 & 119.69 & 17.85 & 173.45 \\
66 & $45 / 45 / 55$ & 72.58 & 120.13 & 167.70 & 174.08 \\
& $45 / 45 / 66$ & 72.43 & 119.80 & 167.34 & 173.55 \\
& $55 / 55 / 55$ & 72.22 & 119.97 & 167.24 & 172.96 \\
78 & $45 / 45 / 55$ & 72.21 & 119.67 & 167.50 & 173.19 \\
& $45 / 45 / 66$ & 72.04 & 119.39 & 167.10 & 172.88 \\
& $55 / 55 / 55$ & 71.88 & 119.06 & 167.00 & 172.43 \\
91 & $45 / 45 / 55$ & 71.82 & 119.23 & 167.18 & 172.33 \\
& $45 / 45 / 66$ & 71.67 & 118.95 & 166.79 & 172.10 \\
& $55 / 55 / 55$ & 71.52 & 118.65 & 166.70 & 171.62 \\
\hline
\end{tabular}

layer plate $\left(\theta=90^{\circ} / 0^{\circ} / 90^{\circ}\right)$ with the following geometrical data

$c / a=g / a=0.25, r / a=0.15, h / a=(0.01 ; 0.1)$

Assume that the plate is made of materials with the following characteristics:

Material I

$\frac{E_{1}}{E_{2}}=10, \frac{G_{12}}{E_{2}}=0.33, \frac{G_{13}}{E_{2}}=0.5, \frac{G_{23}}{E_{2}}=0.5, \nu_{12}=0.22$.

Material II

$\frac{E_{1}}{E_{2}}=40, \frac{G_{12}}{E_{2}}=0.6, \frac{G_{13}}{E_{2}}=0.6, \frac{G_{23}}{E_{2}}=0.5, \nu_{12}=0.25$.

The results of these investigations are presented in Table 3 for the CPT and Table 4 for the FSDT.

The analysis of Table 3 shows that convergence of the frequencies occurs for the 11th degree of the polynomial for the CPT, what corresponds to 78 coordinate functions for deflection $w$ (indeterminate component $\Phi_{3}$ ). Table 4 allows to conclude that, for the FSDT, we can use the approximation of the 12th degree for $w$ (indeterminate component $\Phi_{3}, 91$ coordinate functions), 9th power for the indeterminate component $\Phi_{4}$ (55 coordinate functions) and the 8th degree for indeterminate components $\Phi_{5}, \Phi_{6}$ (45 coordinate functions). Bellow all the results are reported for these numbers of admissible functions.

The effect of the static component of the load $p_{0}$ and the ply orientation on the value of natural frequency parameter $\Lambda_{1}=\Omega_{L} a^{2} \sqrt{\frac{12\left(1-v_{12} \nu_{21}\right)}{E_{2} h^{2}}}$, as well as on the action of the buckling load $p_{c r}=\frac{N_{22} a^{2}}{E_{2} h^{3}}$, can be analyzed using the data reported in Table 5. The presented results have been obtained in the framework of both FSDT and CPT. The results obtained by the FSDT and the CPT correspond to numerators and denominators of fractions, respectively.

An increase in the load component $p_{0}$ results in a decrease in the frequency parameter, which is typical for the most of plate forms, including rectangular ones. Since the plate is thin with the ratio $h / a=0.01$, the results obtained with both theories (CPT and FSDT) are similar.

Below the results for the considered plates with thickness $h=$ $0.1 a$ are presented. These results have been obtained in the framework of the FSDT. Table 6 shows the influence of the load on the values of the natural frequency. Here, the critical load is presented for cross- and angle-ply plates made of Material II (61).

In Table 7 , modes of vibrations and frequency parameters $\Lambda_{i}(i=1,2,3)$, corresponding to an unloaded plate, are presented

\section{Table 5}

Values of the frequency parameter $\Lambda_{1}=\Omega_{L} a^{2} \sqrt{\frac{12\left(1-v_{12 \nu 21)}\right.}{E_{2} h^{2}}}$ and the buckling load $p_{c r}=\frac{N_{22} a^{2}}{E_{2} h^{3}},\left(\frac{F S D T}{C L T}, \frac{h}{a}=0.01\right.$, Material I).

\begin{tabular}{|c|c|c|c|c|c|}
\hline Ply orientation & $p_{c r}$ & $p_{0} / p_{c r}=0 \Lambda_{1}$ & $p_{0} / p_{c r}=0.25 \Lambda_{1}$ & $p_{0} / p_{c r}=0.5 \Lambda_{1}$ & $p_{0} / p_{c r}=0.75 \Lambda_{1}$ \\
\hline $90^{\circ} / 0^{\circ} / 90^{\circ}$ & $\frac{31.68}{29.21}$ & $\frac{71.16}{69.72}$ & $\frac{61.98}{60.77}$ & $\frac{50.97}{50.02}$ & $\frac{36.37}{35.73}$ \\
\hline $15^{\circ} /-15^{\circ} / 15^{\circ}$ & $\frac{14.62}{1256}$ & $\frac{62.09}{56.40}$ & $\frac{55.64}{50.31}$ & $\begin{array}{l}\frac{47.82}{44.97} \\
4.97\end{array}$ & $\begin{array}{l}35.01 \\
33.09\end{array}$ \\
\hline $30^{\circ} /-30^{\circ} / 30^{\circ}$ & $\frac{15.82}{1422}$ & $\frac{62.13}{5798}$ & $\frac{54.77}{51.85}$ & $\frac{45.82}{44.63}$ & $\frac{33.58}{35.51}$ \\
\hline $45^{\circ} /-45^{\circ} / 45^{\circ}$ & $\begin{array}{l}16.22 \\
\frac{16.31}{15.23}\end{array}$ & $\begin{array}{l}5.98 \\
\frac{63.49}{60.42}\end{array}$ & $\begin{array}{l}51.85 \\
55.68 \\
52.87\end{array}$ & $\begin{array}{l}44.63 \\
\frac{46.17}{43.72}\end{array}$ & $\begin{array}{l}35.51 \\
\frac{33.31}{31.40}\end{array}$ \\
\hline
\end{tabular}

Table 6

Values of the frequency parameter $\Lambda_{1}=\Omega_{L} a^{2} \sqrt{\frac{12\left(1-v_{12} \nu_{21}\right)}{E_{2} h^{2}}}$ and the buckling load $p_{c r}=N_{22} a^{2} /\left(E_{2} h^{3}\right),\left(\right.$ FSDT, $\frac{h}{a}=0.1$, Material II).

\begin{tabular}{|c|c|c|c|c|c|}
\hline Ply orientation & $p_{c r}$ & $p_{0} / p_{c r}=0 \Lambda_{1}$ & $p_{0} / p_{c r}=0.25 \Lambda_{1}$ & $p_{0} / p_{c r}=0.5 \Lambda_{1}$ & $p_{0} / p_{c r}=0.75 \Lambda_{1}$ \\
\hline $90^{\circ} / 0^{\circ} / 90^{\circ}$ & 20.73 & 71.00 & 65.77 & 59.92 & 53.03 \\
\hline $15^{\circ} /-15^{\circ} / 15^{\circ}$ & 12.45 & 66.33 & 61.53 & 55.85 & 47.97 \\
\hline $30 /-30 \%$ & 11.71 & 66.84 & 62.19 & 56.91 & 50.61 \\
\hline $45^{\circ} /-45^{\circ} / 45^{\circ}$ & 17.57 & 67.76 & 60.41 & 51.42 & 39.08 \\
\hline
\end{tabular}


Table 7

Modes of vibrations (Material I, $h / a=0.01$ ).
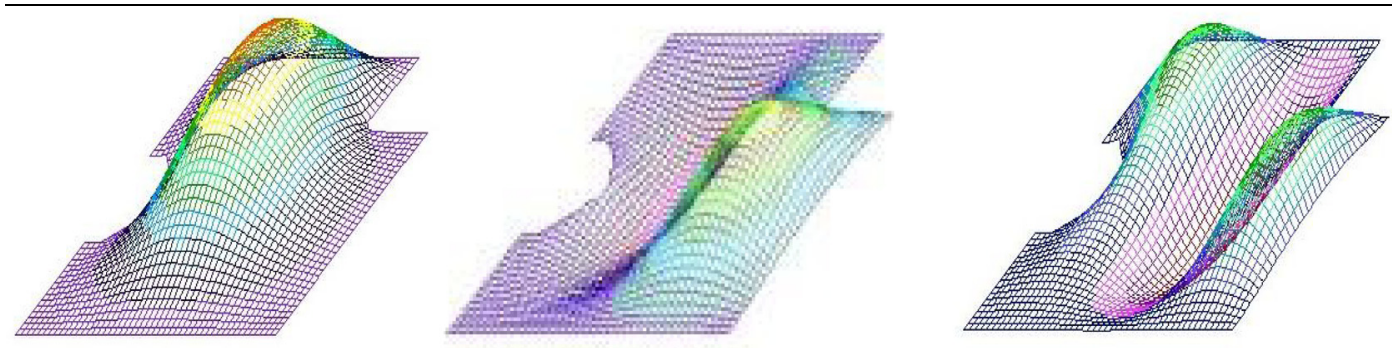

$\Lambda_{1}=69.72$

$\Lambda_{2}=115.66$

$\Lambda_{3}=161.83$
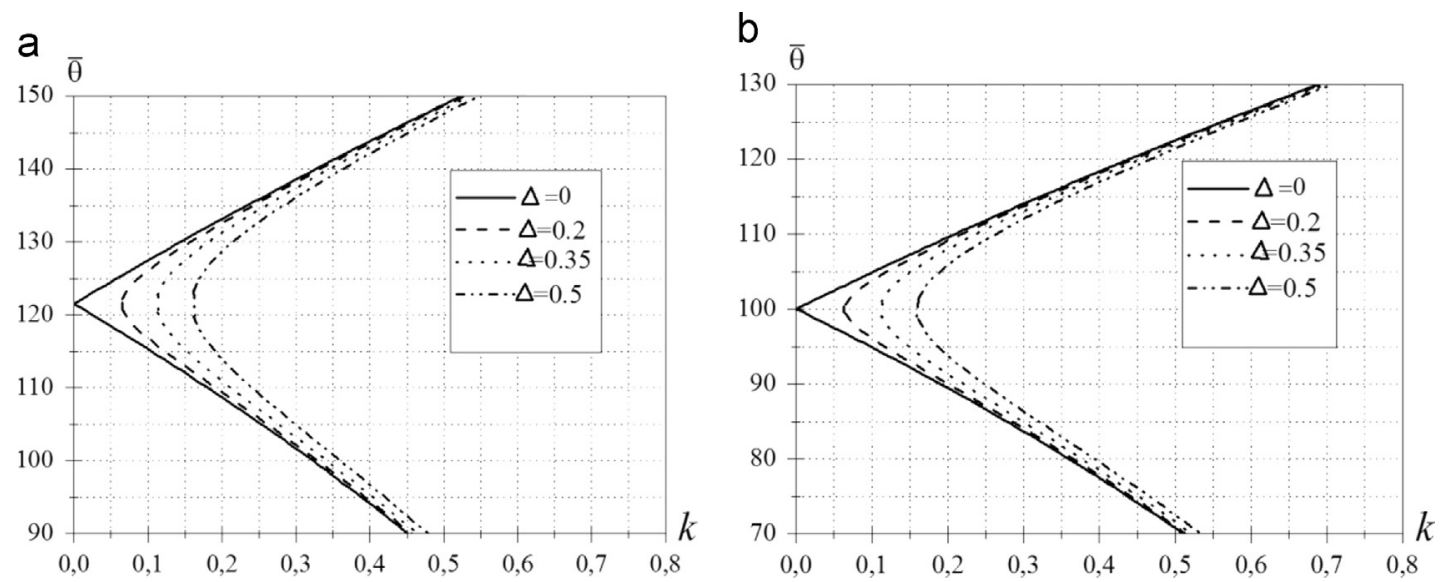

Fig. 6. The effect of damping on instability regions of a cross-ply plate for Material $\mathrm{I}, h / a=0.01$, and obtained by the CPT for $p_{0} / p_{c r}=0.25$ (a) and $p_{0} / p_{c r}=0.5$ (b).

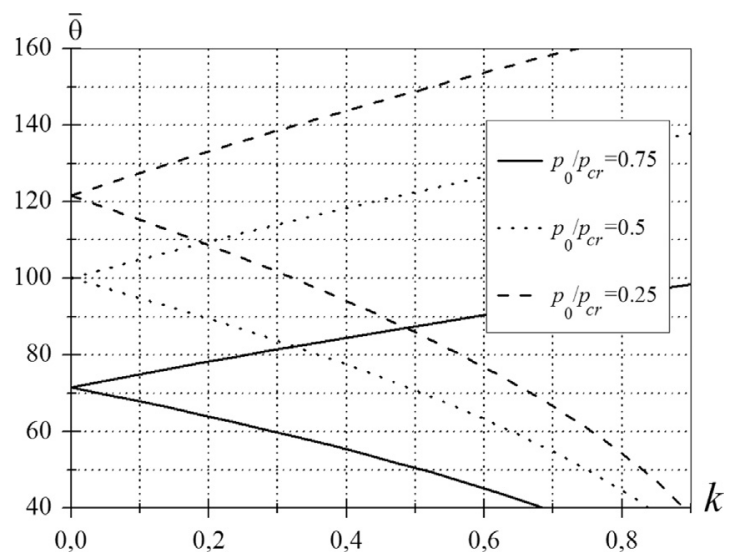

Fig. 7. The effect of the static parameter $p_{0}$ of the load on the instability region of a cross-ply plate for Material I, $h / a=0.01$.

for Material I. The results have been obtained for the cross-ply plate in the framework of CPT.

The instability analysis is performed to study the effect of damping and load parameters on the location of the instability regions. Instability regions constructed for $p_{0} / p_{c r}=0.25,0.5$; $\Delta=0,0.2,0.35,0.5$, Material I, are presented in Fig. 6(a) and (b). It should be emphasized that we have used formulas (26) to

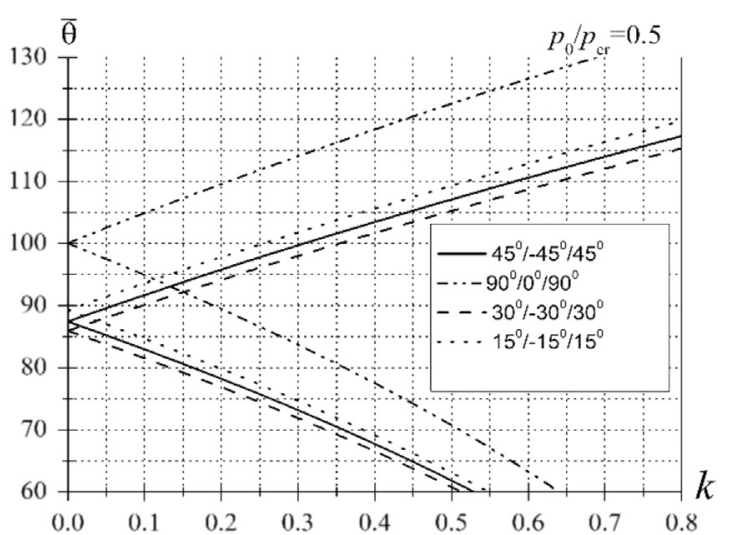

Fig. 8. The effect of the ply orientation on the instability region of a plate for Material I, $h / a=0.01$.

construct instability regions, and the vibration frequency has been replaced by the frequency parameter $\Lambda_{1}$. The analysis of the results allows to conclude that the variation of the damping decrement entails a decrease in the instability zones.

The effect of the static parameter $p_{0}$ on the location of instability zones is shown in Fig. 7. We varied the value of the parameter $p_{0} / p_{c r}=0.75,0.5,0.25$ and other fixed parameters $\left(\Delta=0,90^{\circ} / 0^{\circ} / 90^{\circ}\right)$. With increasing the static component of the 

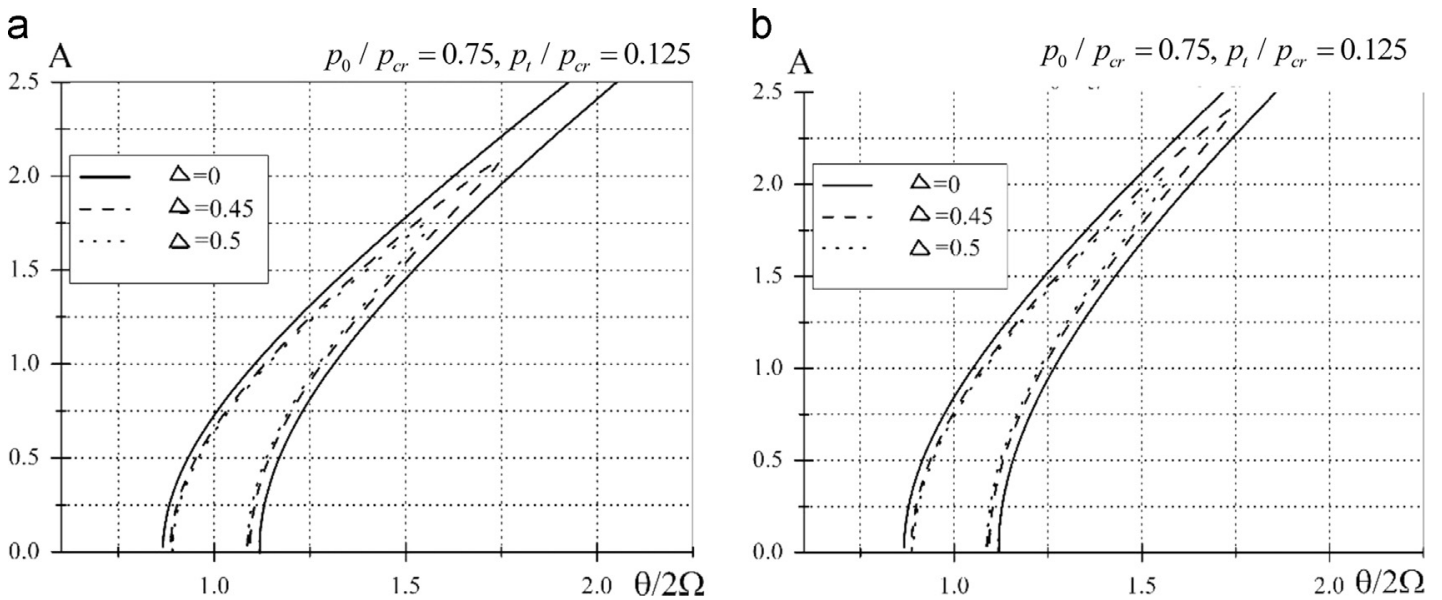

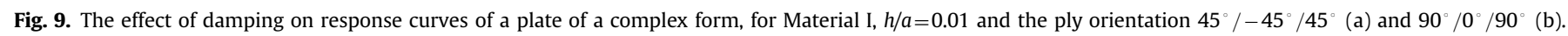
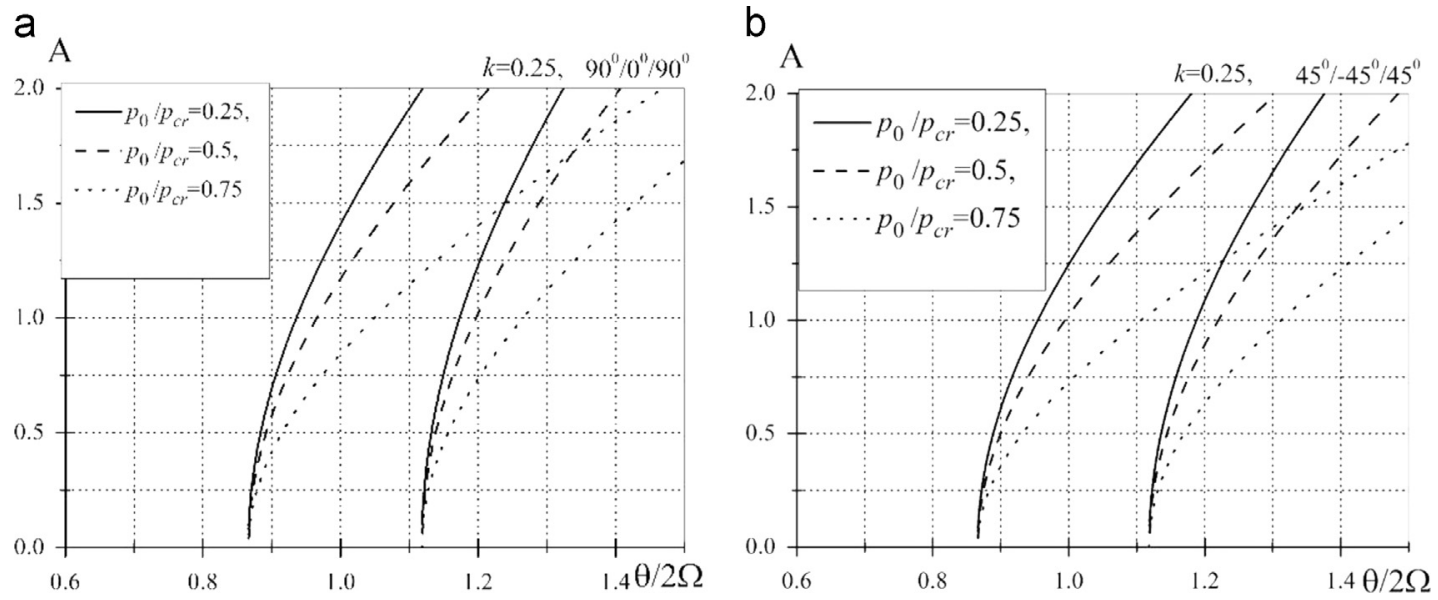

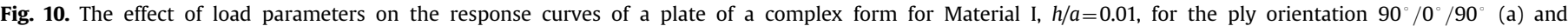
$45^{\circ} /-45^{\circ} / 45^{\circ}$ (b).

load, dynamic instability regions shift towards smaller values of the exciting frequency.

The effect of the ply orientation on instability regions of the considered plate with $p_{0} / p_{c r}=0.5, \Delta=0$ is shown in Fig. 8. We obtain greater frequencies of excitation for the cross-ply plate $\left(90^{\circ} / 0^{\circ} / 90^{\circ}\right)$. On the other hand, instability zones are close to each other in other ply orientations.

For the given plate, non-linear steady vibrations are studied in the main instability region. The effect of damping decrement on amplitude-frequency relations for two types of the ply orientation is shown in Fig. 9. Calculations are performed for $p_{0} / p_{c r}=0.75$, $p_{t} / p_{c r}=0.125$. As it has been expected, the damping factor affects the response curves more significantly for the plates with the ply orientation $45^{\circ} /-45^{\circ} / 45^{\circ}$ than for the cross-ply orientation $\left(90^{\circ} / 0^{\circ} / 90^{\circ}\right)$.

Note that dependence between amplitude $A$ and relations $\frac{\theta}{2 \Omega_{t}}$ has been defined by Bolotin's approach (see formula (41)). In Fig. 9 and in further Figs. 10, 12 and 13, two solutions corresponding to two signs are presented (one of them is unstable). The lower curves describe the unstable solutions. The upper curves, corresponding to processes of increasing the excitation frequency, represent stable solutions. The reported slope of the resonance curve towards higher excitation frequencies is a characteristic feature exhibiting non-linear elasticity.

Amplitude-frequency relations for various values $p_{0} / p_{c r}=$ $0.25,0.5,0.75, \Delta=0, k=0.25$ and two types of the ply orientation: $\left(90^{\circ} / 0^{\circ} / 90^{\circ}\right)$ and $\left(45^{\circ} /-45^{\circ} / 45^{\circ}\right)$ are presented in Fig. 10.

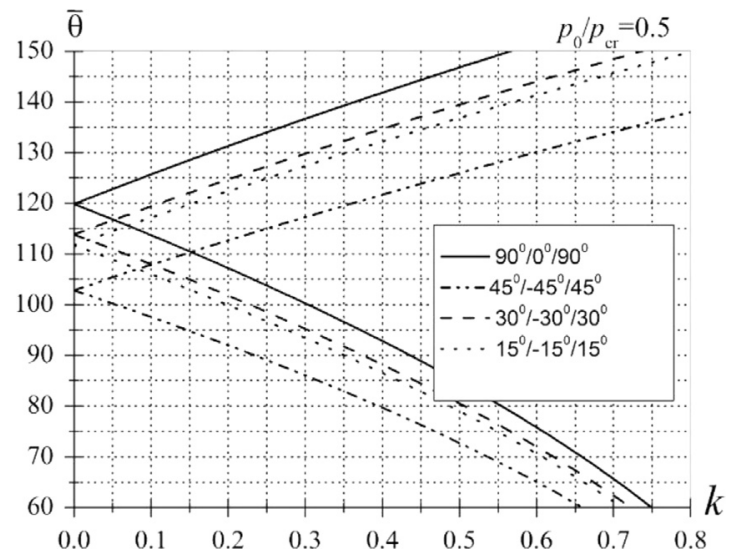

Fig. 11. The effect of the ply orientation on the instability region of a plate for Material II, $h / a=0.1$, obtained by FSDT.

A change of the constant component of the load influences the slope of obtained curves. An increase in $p_{0} / p_{c r}$ results in a decrease in the slope of a tangent to the response curve. For two types of the ply orientation, the response curves are close for $p_{0} / p_{c r}=0.25,0.5$, and differ for $p_{0} / p_{c r}=0.75$.

Further, we study a laminated plate made of Material II with mechanical characteristics given by (61). Geometric parameters 
a

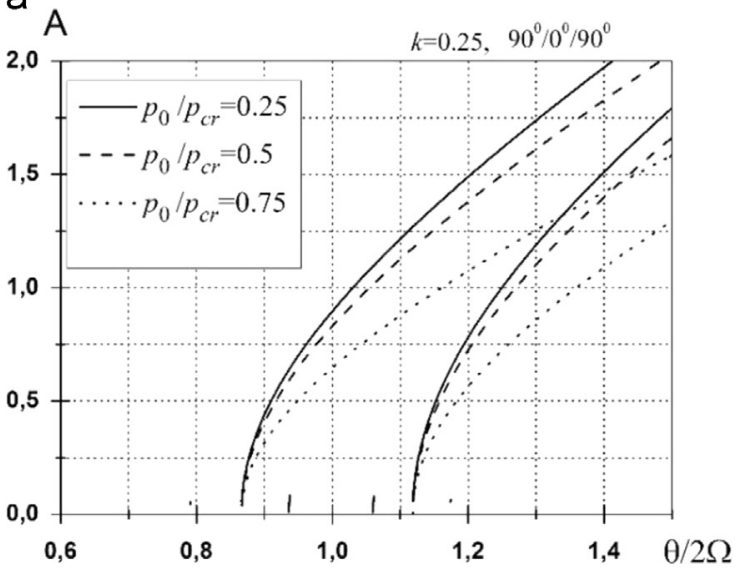

b

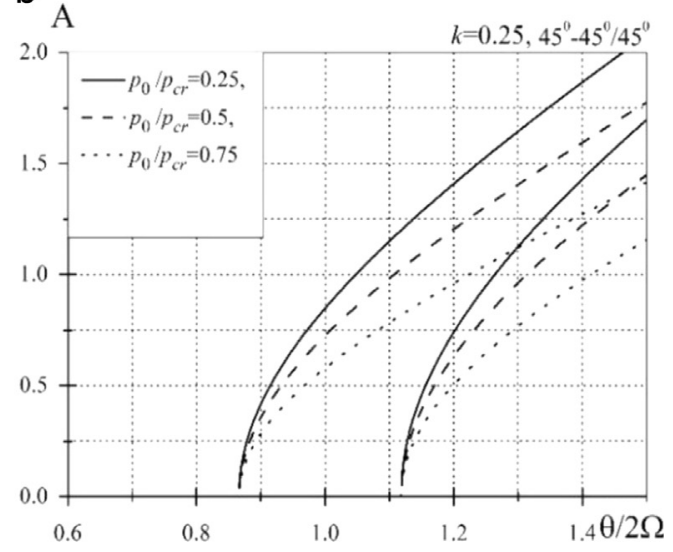

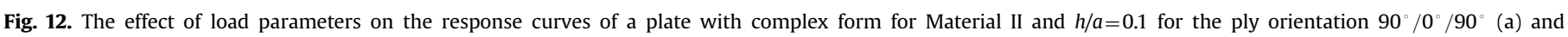
$45^{\circ} /-45^{\circ} / 45^{\circ}$ (b).

are the same as for (59), but $h / a=0.1$. In order to study parametric vibrations of this plate, the FSDT is applied.

Fig. 11 shows instability regions of the considered plate for different types of the ply orientation. Obviously, the location of instability zones for each type of the ply orientation differs more significantly for Material II than for Material I. However, greater frequencies of excitation are again observed for the cross-ply $\left(90^{\circ} \%\right.$ $0^{\circ} / 90^{\circ}$ ) plate.

Response curves for different values of static parameters of the load and for $\Delta=0, k=0.25$, as well as for two types of the ply orientation $\left(90^{\circ} / 0^{\circ} / 90^{\circ}\right)$ and $\left(45^{\circ} /-45^{\circ} / 45^{\circ}\right)$ for the considered plate are presented in Fig. 12. The obtained results demonstrate that for both types of the ply orientation the variation of the constant component of the load affects the slope of a tangent to the response curves.

Fig. 13 shows the effect of the ply orientation on the amplitude-frequency relations of the plate for $p_{0} / p_{c r}=0.5, p_{t} / p_{c r}=0.5$ and Material II. It follows from Fig. 13 that the biggest amplitudes are achieved for the ply orientation $15^{\circ} /-15^{\circ} / 15^{\circ}$.

From the analysis of the results of the investigation we can conclude that the qualitative nature of the behavior of the response curves for plates made of Material I and II and of thicknesses $(h / a=0.01$ and $h / a=0.1)$ is the same when the parameters of the load and damping are changed. However, their instability regions differ if the ply orientation is changed (Figs. 8 and 11).

\section{Concluding remarks}

The present paper introduces the carried-out investigation of parametric vibrations of laminated plates having a complex form and external cutouts. The applied method has been worked out and validated for the classical and the first-order plate theories. The developed method is based on combined application of the Rfunctions theory and variational methods, and may be applied to laminated plates of an arbitrary planform and different boundary conditions, as well as thin and moderately thick plates. A subcritical inhomogeneous state has been taken into account. Due to an original discretization algorithm, the initial system of PDEs has been reduced to ODEs. The analysis of this system by known approaches allows to construct instability zones and amplitudefrequency relations for various parameters of the system. In order to demonstrate the capabilities of the proposed method, a special form of the laminated plate has been chosen. The shape of the plate is complicated owing to the introduction of two non-

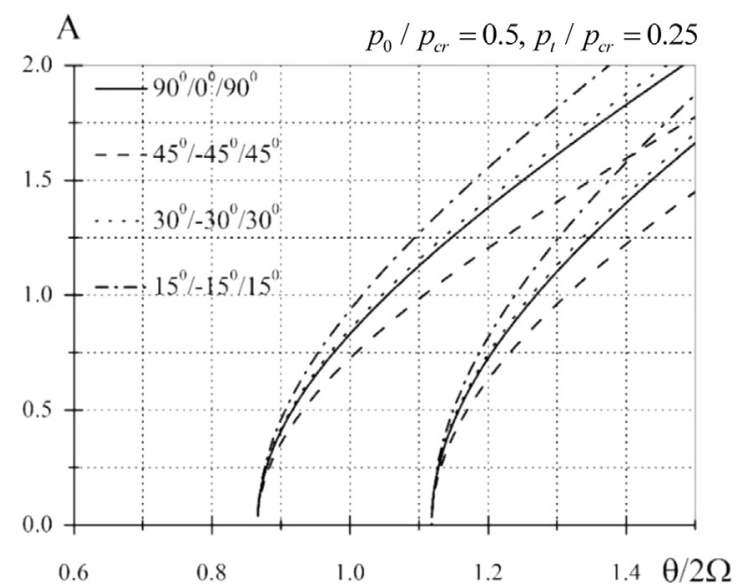

Fig. 13. The effect of the ply orientation on response curves of the plate (Fig. 5) for Material II, $h / a=0.1$.

symmetrically located cutouts of both rectangular and round forms. The main physical feature of this problem concerns loading the plate along one of the sides, and balancing the load by a clamped base. Convergence of the obtained results has been studied. In addition, the influence of different components (static and dynamic load parameters, ply orientation and thickness) on dynamic behavior of the considered plate has been investigated.

We have proposed an algorithm for transformation of PDEs to a corresponding system of ODEs, taking into account both multimode approximations and formulas (33), (34) for calculations of the coefficients of this system. In other words, we have theoretically solved the stated problem in full. Only the presented numerical results concern a single-mode approximation. However, the so far obtained results are validated through a comparison to those obtained in other researches, which gives a hope to solve numerous problems related to modes interaction within the applied FSDT method, having in mind the already proposed theoretical background.

\section{Acknowledgments}

This paper was financially supported by the National Science Centre of Poland under the Grant MAESTRO 2, No. 2012/04/A/ST8/ 00738, for years 2013-2016. 


\section{References}

[1] M. Amabili, Nonlinear Vibrations and Stability of Shells and Plates, Cambridge University Press, Cambridge, 2008.

[2] V. Anil, C.S. Upadhyay, N.G.R. Iyengar, Stability analysis of composite laminate with and without rectangular cutout under biaxial loading, Compos. Struct. 80 (2007) 92-104.

[3] J. Awrejcewicz, L. Kurpa, O. Mazur, On the parametric vibrations and meshless discretization of orthotropic plates with complex shape, Int. J. Nonlinear Sci. Numer. Simul. 11 (5) (2010) 371-386.

[4] V.V. Bolotin, Dynamic Stability of Elastic Systems, Holden-Day, San Francisco, 1964.

[5] L.W. Chen, J.Y. Yang, Dynamic stability of laminated composite plate by the finite element method, Comput. Struct. 36 (1990) 845-851.

[6] S. Dash, A.V. Asha, S.K. Sahu, Stability of laminated composite curved panels with cutout using finite element method, in: Proceedings of the International Conference on Theoretical, Applied Computational and Experimental Mechanics, ICTACEM 2004, 28-31 December, IIT, Kharagpur, 2004.

[8] P.K. Datta, S. Biswas, Research advances on tension buckling behavior of aerospace structures: a review, Int. J. Aeronaut. Space Sci. 12 (1) (2011) 1-15.

[10] A. Koushal, R.B. Bhat, A comparison study of vibration of plate with cutouts using the finite element and Rayleigh-Ritz methods, in: Proceedings of Canadian Congress of Applied Mechanics, CANCAM'93, Ontario, 1993.

[11] L.V. Kurpa, R-functions Method for Solving Linear Problems of Bending and Vibrations of Shallow Shells, Kharkiv NTU Press, Kharkiv, 2009 (in Russian).

[12] L. Kurpa, O. Mazur, V. Tkachenko, Dynamical stability and parametrical vibrations of the laminated plates with complex shape, Lat. Am. J. Solids Struct. 10 (2013) 175-188.

[13] L.V. Kurpa, O.S. Mazur, V.V. Tkachenko, Parametric vibrations of multilayer plates of complex shape, J. Math. Sci. 174 (2) (2014).

[15] H.P. Lee, S.P. Lim, S.T. Chow, Prediction of natural frequencies of rectangular plates with rectangular cutouts, Comput. Struct. 36 (1990) 861-869.

[16] Michael P. Nemeth, Buckling and postbuckling behavior of laminated composite plates with a cutout, Nasa technical paper 3587, 1996.

[17] G. Mundkur, R.B. Bhat, S. Neriya, Vibration of plates with cutouts using boundary characteristics orthogonal polynomial functions in the Rayleigh-Ritz method, J. Sound Vib. 176 (1994) 136-144.

[18] N.S. Putcha, J.N. Reddy, Stability and natural analysis of laminated plates by using a mixed element based on a refined plate theory, J. Sound Vib. 104 (2) (1986) 285-300.

[19] M.S. Qatu, R.W. Sullivan, W. Wang, Recent research advances on the dynamic analysis of composite shells: 2000-2009, Compos. Struct. 93 (1) (2010) 14-31.

[20] J.N. Reddy, Mechanics of Laminated Composite Plates and Shells. Theory and Analysis, Second ed., CRC PRESS, London, 2004.

[21] J.N. Reddy, N.D. Phan, Stability and vibration of isotropic, orthotropic and laminated plates according to a higher-order shear deformation theory, J. Sound Vib. 98 (2) (1985) 157-170.

[22] V.L. Rvachev, The R-Functions Theory and its Some Application, Naukova Dumka, Kiev, 1982 (in Russian).

[23] S.K. Sahu, P.K. Datta, Dynamic stability of laminated copmposite curved panels with cutouts, J. Eng. Mech. 129 (11) (2003) 1245-1253.
[24] S.K. Sahu, P.K. Datta, Dynamic stability of curved panels with cutouts, J. Sound Vib. 251 (4) (2002) 683-696.

[25] S.K. Sahu, P.K. Datta, Research advances in the dynamic stability behavior of plates and shells: 1987-2005. Part1: conservative system, Appl. Mech. Rev. 60 (2007) 65-75.

[26] Y.X. Zhang, C.H. Yang, Recent developments in finite element analysis for laminated composite plates, Compos. Struct. 88 (1) (2009) 147-157.

[27] W.R. Chen, C.S. Chen, J.H. Shyu, Stability of parametric vibrations of laminated composite plates, Appl. Math. Comput. 223 (2013) 127-138.

[28] K.L. Turner, S.A. Miller, P.G. Hartwell, N.C. McDonald, S.H. Strogatz, S.G. Adams, Five parametric resonances in a microelectromechanical system, Nature 396 (1998) 149-152.

29] D.W. Carr, S. Evoy, L. Sekaric, H.G. Craghead, J.M. Parpia, Parametric amplification in a torsional microresonator, Appl. Phys. Lett. 77 (10) (2000) 1545-1549.

30] W. Zhang, R. Baskaran, K.L. Turner, Effect of cubic nonlinearity on autoparametrically amplified resonant MEMS mass sensor, Sensors Actuators A 102 (2002) 139-150.

[31] D.V. Scheible, A. Erbe, R.H. Blick, Evidence of a nanomechanical resonator being driven into chaotic response via the Ruelle-Takens route, Appl. Phys. Lett. 81 (10) (2002) 1884-1890.

[32] W. Zhang, G. Meng, Nonlinear dynamical ssystem of micro-cantilever under combined parametric and forcing excitations in MEMS, Sensors Actuators A 119 (2005) 291-299.

[33] B.E. DeMartini, J.F. Rhoads, K.L. Turner, S.W. Shaw, J. Moehlis, Linear and nonlinear tuning of parametrically excited MEMS oscillators, J. Microelec tromech. Syst. 16 (2) (2007) 310-315.

[34] B.E. DeMartini, H.E. Butterfield, J. Moehlis, K.L. Turner, Chaos for a microelectromechanical oscillator governed by the nonlinear Mathieu equation, J. Microelectromech. Syst. 16 (6) (2007) 314-319.

[35] M.I. Younis, E.M. Abdel-Rahman, A reduced-order model for electrically actuated microbeam-based MEMS, J. Microelectromech. Syst. 12 (5) (2003) 672-678.

[36] J. Awrejcewicz, Ordinary Differential Equations and Mechanical Systems, Springer, Berlin, 2014

\section{Further reading}

[7] P.K. Datta, An investigation of the buckling behavior and parametric resonance phenomenon of a tensioned sheet with a central opening, J. Sound Vib. 58 (4) (1978) 527-534.

9] M. Ganapathi, P. Boisse, D. Solaut, Non-linear dynamic stability analysis of composite laminates under periodic in-plane compressive loads, Int. J. Numer. Methods Eng. 46 (1999) 943-956.

[14] K.Y. Lam, K.C. Hung, Orthogonal polynomials and subsectioning method for vibration of plates, Comput. Struct. 34 (1990) 827-834. 